\title{
Enumeration of some matrices and free linear codes over commutative finite local rings
}

https://doi.org/10.1515/spma-2021-0150

Received July 18, 2021; accepted October 4, 2021

Abstract: Let $R$ be a commutative finite local ring. Two enumeration problems over $R$ are presented. We enumerate the matrices over $R$ with a given McCoy rank and a given number of rows of single unit, and the free linear codes over $R$ which have a given rank and a given number of vectors of single unit.

Keywords: McCoy rank, free linear code, local ring

MSC: 15A03, 94B05

\section{Introduction}

Problems concerning the enumeration of matrices over finite fields under given conditions have been classically studied. A well-known basic formula gives the number of $m \times n$ matrices over the finite field $\mathbb{F}_{q}$ of order $q$ of rank $r$ :

$$
\prod_{i=0}^{r-1} \frac{\left(q^{m}-q^{i}\right)\left(q^{n}-q^{i}\right)}{q^{r}-q^{i}},
$$

see [8] for the proof. Abdel-Ghaffar [1] analyzed matrices over $\mathbb{F}_{q}$ with rows of single unit over $\mathbb{F}_{q}$, i.e. rows that have a single nonzero entry. $N_{\mathbb{F}_{q}}(m, n, r, k)$, then denotes the number of $m \times n$ matrices over $\mathbb{F}_{q}$ of rank $r$ that have exactly $k$ rows of single unit. This is discussed in [1], and is equal to

$$
\sum_{i=k}^{m}(-1)^{i-k}\left(\begin{array}{c}
i \\
k
\end{array}\right)\left(\begin{array}{c}
m \\
i
\end{array}\right) \sum_{\ell=0}^{r}\left(\begin{array}{l}
n \\
\ell
\end{array}\right) \ell ! S(i, \ell)(q-1)^{i} q^{(m-i) \ell} \prod_{j=0}^{r-\ell-1} \frac{\left(q^{m-i}-q^{j}\right)\left(q^{n-\ell}-q^{j}\right)}{q^{r-\ell}-q^{j}}
$$

where $S(i, \ell)$ is the Stirling number of the second kind, or the number of ways in which a set of $i$ elements can be divided into $\ell$ nonempty subsets. Note that

$$
\begin{aligned}
& S(0,0)=1, S(i, 0)=0 \text { if } i>0, S(i, \ell)=0 \text { if } i<\ell \\
& S(i, \ell)=\frac{1}{\ell !} \sum_{j=0}^{\ell}(-1)^{\ell-j}\left(\begin{array}{l}
\ell \\
j
\end{array}\right) t^{i} \text { if } i \geq 1 \text { and } i \geq \ell .
\end{aligned}
$$

Abdel-Ghaffar showed that this may be folded into coding theory by counting the number of linear codes of $\mathbb{F}_{q}^{n}$ that contain a given number of vectors of single unit. At the same time, the number of linear codes containing no vectors of single unit is also obtained. This is useful in error detection and correction, since the minimum distance of a linear code containing no vectors of single unit is at least two (see [10]).

We know that all nonzero elements in a field are units. However, a commutative ring which is not a field consists of many zero divisors and units. This typically makes working over rings more difficult than working

^Corresponding Author: Siripong Sirisuk: Department of Mathematics and Statistics, Faculty of Science and Techonology, Thammasat University, Phathum Thani 12120, Thailand, E-mail: siripong@mathstat.sci.tu.ac.th, siripong.srs@gmail.com 
over fields. Nevertheless, over the past decades, much has been understood about matrices and codes over rings [2], [3], [4], [6], [7], and [13]. The purpose of this paper is to generalize the results of [1] on matrices and linear codes over finite fields to commutative finite local rings.

\section{Preliminaries}

Throughout the paper, our rings contain the identity $1 \neq 0$.

Let $R$ be a finite commutative ring. A row vector $\vec{r}=\left(r_{1}, r_{2}, \ldots, r_{n}\right) \in R^{n}$ is called a row of single unit or vector of single unit if a single entry is a unit and all other entries are zero. It is clear that, if $R$ is a field, then a row of single unit is a row having a single nonzero entry. For $i=1,2, \ldots, n$, let $\vec{e}_{i}=\left(e_{i 1}, e_{i 2}, \ldots, e_{i n}\right) \in R^{n}$ where $e_{i i}=1$ and $e_{i j}=0$ for all $i \neq j$. Clearly, $\vec{e}_{1}, \vec{e}_{2}, \ldots, \vec{e}_{n}$ are vectors of single unit which form a basis of the $R$-module $R^{n}$. They are therefore known as standard basis vectors. Moreover, $\vec{x}$ is a vector of single unit if and only if $\vec{x}=u \vec{e}_{i}$ for some unit $u \in R$ and standard vector $\vec{e}_{i}$. A set $\left\{\vec{x}_{1}, \vec{x}_{2}, \ldots, \vec{x}_{r}\right\}$ of vectors in $R^{n}$ is said to be linearly independent, if for any $a_{1}, a_{2}, \ldots, a_{r}$ in $R, a_{1} \vec{x}_{1}+a_{2} \vec{x}_{2}+\ldots a_{r} \vec{x}_{r}=\overrightarrow{0}$ implies that $a_{1}=a_{2}=\cdots=a_{r}=0$. It is clear that a set of standard basis vectors is linearly independent. A submodule $C$ of the $R$-module $R^{n}$ generated by a linearly independent set of $r$ vectors is called a free linear code of rank $r$, and this linearly independent set is called a basis for $C$.

Next, we introduce McCoy's concept of rank of matrices over commutative rings [11]. This generalizes the rank of matrices over fields. Let $A$ be an $m \times n$ matrix over $R$. For $1 \leq t \leq \min \{m, n\}$, let $I_{t}(A)$ be the ideal of $R$ generated by the $t \times t$ minors of $A$ and $I_{0}(A)=R$. It follows that

$$
\{0\}=\operatorname{Ann}_{R} I_{0}(A) \subseteq \operatorname{Ann}_{R} I_{1}(A) \subseteq \cdots \subseteq \operatorname{Ann}_{R} I_{\min \{m, n\}}(A),
$$

where $\operatorname{Ann}_{R} I=\{r \in R: r b=0$ for all $b \in I\}$ is the annihilator of $I$. The McCoy rank or rank of $A$, denoted $\operatorname{rank} A$, is the maximum number $r$ such that $\operatorname{Ann}_{R} I_{r}(A)=\{0\}$. If $R$ is a field, this rank coincides with the usual rank. Some properties of this rank over commutative rings resemble those of the rank over fields. For instance, an $n \times n$ invertible matrix over $R$ is of rank $n$. The set of $n \times n$ invertible matrices over $R$ is called the general linear group of degree $n$, denoted by $G L_{n}(R)$. Next, let $A$ be an $m \times n$ matrix over $R$. Then $0 \leq \operatorname{rank} A \leq \min \{m, n\}$, $\operatorname{rank} A=\operatorname{rank} A^{T}, \operatorname{rank} A=\operatorname{rank} P A Q$ for all $P \in G L_{m}(R)$ and $Q \in G L_{n}(R)$, and $\operatorname{rank} A=0$ if and only if $\operatorname{Ann}_{R} I_{1}(A) \neq\{0\}$. Moreover, when $m=n$, rank $A<n$ if and only if det $A$ is the zero or a zero divisor of $R$, and when $m \leq n, A$ has rank $m$ if and only if the rows of $A$ are linearly independent. References [4] and [5] set out further properties. Furthermore, the rank of a matrix over $R$ is simple to compute when $R$ is a commutative finite local ring.

A local ring is a ring with unique maximal ideal. Clearly, a field is a commutative local ring with unique maximal ideal $\{0\}$. If $M$ is the maximal ideal of a commutative local ring $R$, then $R \backslash M$ is the group of units of $R$ and the quotient ring $R / M$ is a field that is known as the residue field of $R$. A commutative local ring $R$ is also equipped with the natural map $\pi: R \rightarrow R / M$, given by $\pi(a)=a+M$ for all $a \in R$. Hence, if $A=\left[a_{i j}\right]$ is a matrix over $R$, then $\pi(A)=\left[\pi\left(a_{i j}\right)\right]$ is a matrix over its residue field $R / M$. The rank of matrices over commutative finite local rings can be computed by the following lemma.

Lemma 2.1. [3] Let $R$ be a commutative finite local ring with maximal ideal $M$ and the natural map $\pi: R \rightarrow$ $R / M$. If $A$ is a matrix over $R$, then $\operatorname{rank} A=\operatorname{rank} \pi(A)$.

In the rest of this paper, we first determine the number of $m \times n$ matrices over a commutative finite local ring of rank $r$. Among these, we count the number of matrices with a given number of rows of single unit. Relationships of such matrices over a commutative finite local ring and matrices over its residue field are discussed. We then note some properties of free linear codes over commutative finite local rings. Relationships of free linear codes with vectors of single unit and matrices with rows of single unit are discussed. Finally, we present a formal expression of the number of free linear codes of rank $r$ containing a given number of standard basis vectors. 


\section{Main Results}

\subsection{Matrices with rows of single unit}

In this section we enumerate $m \times n$ matrices over a commutative finite local ring $R$ of rank $r$. To do this we apply lifting.

Proposition 3.1. Let $R$ be a commutative finite local ring with maximal ideal $M$, residue field $R / M$ of $q$ elements, and the natural map $\pi: R \rightarrow R / M$.

(1) If $A=\left[a_{i j}\right]$ is a matrix over $R$ such that $\pi(A)=\left[\pi\left(a_{i j}\right)\right]$ is a matrix over $R / M$ of rank $r$, then $\left[a_{i j}+m_{i j}\right]$ is a matrix over $R$ of rank $r$ for all $m_{i j} \in M$.

(2) The number of $m \times n$ matrices over $R$ of rank $r$ is given by

$$
N_{R}(m, n, r)=|M|^{m n} \prod_{i=0}^{r-1} \frac{\left(q^{m}-q^{i}\right)\left(q^{n}-q^{i}\right)}{q^{r}-q^{i}} .
$$

Proof. Note that if $A$ is a matrix over $R$ of rank $r$ then, by Lemma 2.1, $\pi(A)$ is a matrix over $R / M$ of rank $r$. To prove (1), suppose that $\pi(A)=\left[\pi\left(a_{i j}\right)\right]$ is an $m \times n$ matrix over $R / M$ of rank $r$. Let $m_{i j} \in M$ for all $i=1,2, \ldots, m$ and $j=1,2, \ldots, n$. Hence, $\operatorname{rank}\left(\left[a_{i j}+m_{i j}\right]\right)=\operatorname{rank}\left(\pi\left[a_{i j}+m_{i j}\right]\right)=\operatorname{rank}\left(\left[\pi\left(a_{i j}+m_{i j}\right)\right]\right)=\operatorname{rank}\left[\pi\left(a_{i j}\right)\right]=r$.

When applying the formula in (1.1) over a finite field, the number of $m \times n$ matrices over the residue field $R / M$ of rank $r$ is equal to

$$
\prod_{i=0}^{r-1} \frac{\left(|R / M|^{m}-|R / M|^{i}\right)\left(|R / M|^{n}-|R / M|^{i}\right)}{|R / M|^{r}-|R / M|^{i}} .
$$

From (1), we see that each matrix over $R / M$ of rank $r$ can be lifted to $|M|^{m n}$ matrices over $R$ of rank $r$. Thus, the number of $m \times n$ matrices over $R$ of rank $r$ is

$$
|M|^{m n} \prod_{i=0}^{r-1} \frac{\left(|R / M|^{m}-|R / M|^{i}\right)\left(|R / M|^{n}-|R / M|^{i}\right)}{|R / M|^{r}-|R / M|^{i}} .
$$

Since $|R / M|=q$, we have (2).

We next consider the relationship between rows of single unit over a commutative finite local ring and those over its residue field.

Lemma 3.2. Let $R$ be a commutative finite local ring with maximal ideal $M$ and the natural map $\pi: R \rightarrow R / M$. Suppose that $\vec{a}=\left(a_{1}, a_{2}, \ldots, a_{n}\right)$ is a row of a matrix $A$ over $R$.

(1) If $\vec{a}$ is a row of single unit of $A$, then $\pi(\vec{a})$ is a row of single unit of $\pi(A)$.

(2) If $\pi(\vec{a})=\left(M, \ldots, M, a_{i}+M, M, \ldots, M\right)$ is a row of single unit of $\pi(A)$, then

$$
\left(0, \ldots, 0, a_{i}+m_{i}, 0, \ldots, 0\right)
$$

is a row of single unit of $A$ for all $m_{i} \in M$.

Proof. (1) If $\vec{a}$ is a row of single unit of $A$, then there exists $i \in\{1,2, \ldots, n\}$ such that $a_{i}$ is a unit in $R$ and $a_{j}=0$ for all $j \neq i$. This implies that $\pi(\vec{a})=\left(M, \ldots, M, a_{i}+M, M, \ldots, M\right)$ is a row of single unit of $\pi(A)$.

(2) Assume that $\pi(\vec{a})=\left(M, \ldots, M, a_{i}+M, M, \ldots, M\right)$ is a row of single unit of $\pi(A)$. Then $a_{i}$ is a unit in $R$. Thus, $\left(0, \ldots, 0, a_{i}+m_{i}, 0, \ldots, 0\right)$ is a row of single unit of $A$ for all $m_{i} \in M$.

We next derive the number of $m \times n$ matrices of rank $r$ having exactly $k$ rows of single unit. 
Theorem 3.3. Let $R$ be a commutative finite local ring with maximal ideal $M$, residue field $R / M$, and the natural map $\pi: R \rightarrow R / M$.

(1) If $A$ is an $m \times n$ matrix over $R$ of rank $r$ with $k$ rows of single unit, then $\pi(A)$ is a matrix over $R / M$ of rank $r$ with $k$ rows of single unit.

(2) A matrix over $R / M$ of rank $r$ with $k$ rows of single unit can be lifted to $|M|^{m n-n k+k}$ matrices over $R$ of rank $r$ with $k$ rows of single unit.

(3) The number of $m \times n$ matrices over $R$ of rank $r$ having exactly $k$ rows of single unit is given by

$$
|M|^{m n-n k+k} N_{R / M}(m, n, r, k),
$$

where $N_{R / M}(m, n, r, k)$ is the number given in (1.2).

Proof. (1) Let $A$ be an $m \times n$ matrix over $R$ of rank $r$ with $k$ rows of single unit. It follows from Proposition 3.1 and Lemma $3.2(1)$ that $\pi(A)$ is a matrix over $R / M$ of rank $r$ with $k$ rows of single unit.

(2) Let $\left[a_{i j}+M\right]$ be an $m \times n$ matrix over $R / M$ of rank $r$ with $k$ rows of single unit. Without loss of generality, we suppose that the first $k$ rows are rows of single unit. Then there exist $j_{1}, j_{2}, \ldots, j_{k} \in\{1,2, \ldots, n\}$ such that $a_{1 j_{1}}, a_{2 j_{2}}, \ldots, a_{k j_{k}}$ are units in $R$. By Lemma 3.2 (2), for each $i \in\{1,2, \ldots, k\},\left(0, \ldots, 0, a_{i j_{i}}+m_{i j_{i}}, 0, \ldots, 0\right)$ is a row of single unit for all $m_{i j_{i}} \in M$. Thus,

$$
A=\left[\begin{array}{ccccc}
0 & a_{1 j_{1}}+m_{1 j_{1}} & 0 & \cdots & 0 \\
\vdots & & & & \vdots \\
0 & 0 & a_{k j_{k}}+m_{k j_{k}} & \cdots & 0 \\
a_{k+1,1}+m_{k+1,1} & a_{k+1,2}+m_{k+1,2} & \cdots & \cdots & a_{k+1, n}+m_{k+1, n} \\
\vdots & & & & \vdots \\
a_{m 1}+m_{m 1} & a_{m 2}+m_{m 2} & \cdots & \cdots & a_{m n}+m_{m n}
\end{array}\right]
$$

is an $m \times n$ matrix with $k$ rows of single unit for all $m_{i j} \in M$, where $i, j \in\{1,2, \ldots, n\}$. Moreover, $\pi(A)=$ $\left[a_{i j}+M\right]$. Since $\operatorname{rank}\left[a_{i j}+M\right]=r$, Lemma 2.1 implies that $\operatorname{rank} A=r$. Hence, the matrix $\left[a_{i j}+M\right]$ is lifted to $|M|^{m n-n k+k}$ matrices over $R$ of rank $r$ with $k$ rows of single unit.

Note that the number of $m \times n$ matrices over $R / M$ of rank $r$ having exactly $k$ rows of single unit is $N_{R / M}(m, n, r, k)$. All these matrices may be lifted to $|M|^{m n-n k+k}$ matrices over $R$ of rank $r$ with $k$ rows of single unit. This yields (3).

Such matrices over commutative finite local rings, which have rows of single unit, are related to certain free linear codes.

\subsection{Free linear codes and standard basis vectors}

Free linear codes over rings are an active research field. Dougherty and Saltürk [7] determined the number of free linear codes of $R^{n}$ of rank $r$ when $R$ is a Frobenius commutative finite local ring. Sirisuk and Meemark [12] determined the number of free linear codes of $R^{n}$ of rank $r$ when $R$ is an arbitrary commutative finite local ring. Both are given by

$$
|M|^{n r-r^{2}}\left[\begin{array}{l}
n \\
r
\end{array}\right]_{q}
$$

where $\left[\begin{array}{l}n \\ r\end{array}\right]_{q}$ is the number of linear codes of $\mathbb{F}_{q}^{n}$ of dimension $r$ given by

$$
\left[\begin{array}{l}
n \\
r
\end{array}\right]_{q}=\frac{\left(q^{n}-1\right)\left(q^{n}-q\right) \cdots\left(q^{n}-q^{r-1}\right)}{\left(q^{r}-1\right)\left(q^{r}-q\right) \cdots\left(q^{r}-q^{r-1}\right)}=\left[\begin{array}{c}
n \\
n-r
\end{array}\right]_{q} .
$$


When $R$ is the finite field $\mathbb{F}_{q}$ of order $q$, it was shown in [1] that the number of linear codes of $\mathbb{F}_{q}^{n}$ of dimension $r$ containing exactly $k$ standard basis vectors is given by

$$
\sum_{\ell=k}^{r}(-1)^{\ell-k}\left(\begin{array}{l}
\ell \\
k
\end{array}\right)\left(\begin{array}{l}
n \\
\ell
\end{array}\right)\left[\begin{array}{l}
n-\ell \\
n-r
\end{array}\right]_{q}
$$

To generalize this to a commutative finite local ring $R$, we count the number of free linear codes of $R^{n}$ of rank $r$ that contain exactly $k$ standard basis vectors.

Let $C$ be a free linear code of $R^{n}$ of rank $r$ with basis $\mathcal{B}=\left\{\vec{b}_{1}, \vec{b}_{2}, \ldots, \vec{b}_{r}\right\}$. An $r \times n$ matrix $G$ whose rows form a basis for $C$ is called a generator matrix for $C$, that is,

$$
G=\left[\begin{array}{c}
\vec{b}_{1} \\
\vec{b}_{2} \\
\vdots \\
\vec{b}_{r}
\end{array}\right] .
$$

Since the rows of $G$ are linearly independent, it follows that $\operatorname{rank} G=r,|C|=|R|^{r}$ and $C=\left\{\vec{a} G: \vec{a} \in R^{r}\right\}$. If, on the other hand, $C$ is a free linear code of rank $r$ and $\vec{x}_{1}, \vec{x}_{2}, \ldots, \vec{x}_{r}$ are vectors in $C$ such that the $r \times n$ matrix $\left[\begin{array}{c}\vec{x}_{1} \\ \vdots \\ \vec{x}_{r}\end{array}\right]$ is of rank $r$, then $\left\{\vec{x}_{1}, \vec{x}_{2}, \ldots, \vec{x}_{r}\right\}$ is linearly independent, and provides a basis for $C$. In addition, if $G_{1}$ and $G_{2}$ are both generator matrices for $C$, then $G_{1}=U G_{2}$ where $U \in G L_{r}(R)$. Two free linear codes are said to be equivalent if and only if one can be obtained from the other by permuting the coordinates.

Next, we give the forms of generator matrices of free linear codes over commutative finite local rings. We invoke the following lemma.

Lemma 3.4. [12] Let $R$ be a commutative finite local ring and $\vec{a}$ a vector in $R^{n}$. Then $\vec{a}$ is linearly independent if and only if $\vec{a}$ has a coordinate with a unit.

Proposition 3.5. Let $R$ be a commutative finite local ring. Assume that $C$ is a free linear code of $R^{n}$ of rank $r$. Then $C$ is equivalent to a free linear code with a generator matrix $\left[\begin{array}{ll}I_{r} & A\end{array}\right]$, where $A$ is an $r \times(n-r)$ matrix over $R$. Moreover, if $C$ contains at least $k$ standard basis vectors, then $C$ is equivalent to a free linear code with $a$ generator matrix

$$
\left[\begin{array}{ccc}
I_{k} & 0 & 0 \\
0 & I_{r-k} & A^{\prime}
\end{array}\right]
$$

where $A^{\prime}$ is an $(r-k) \times(n-r)$ matrix over $R$.

Proof. Let $C$ be a free linear code of $R^{n}$ of rank $r$ with a generator matrix $G$. We perform row operations on $G$. Since the first row of $G$ is linearly independent it has, by Lemma 3.4, a coordinate with a unit. We can multiply the first row by the inverse of this unit so that the coordinate becomes 1 . We then use the 1 in the first row to force the entries in all other rows within that coordinate to 0 . This yields a new generator matrix $U G$ where $U \in G_{r}(R)$. The rows of $U G$ form a basis for $C$, and thus are linearly independent. We then apply the same process to the second and subsequent rows. The new generator matrix $G^{\prime}$ obtained for $C$ is equivalent to a matrix of the form $\left[\begin{array}{ll}I_{r} & A\end{array}\right]$ for some $r \times(n-r)$ matrix $A$ after permuting the columns of $G^{\prime}$. Therefore, $C$ is equivalent to a free linear code with a generator matrix $\left[\begin{array}{ll}I_{r} & A\end{array}\right]$.

Next, without loss of generality, we may assume that $C$ is a free linear code of rank $r$ generated by $\left[\begin{array}{ll}I_{r} & A\end{array}\right]$, and contains at least $k$ standard basis vectors. Suppose that $\vec{e}_{i}$ are in $C$ where $i>r$. Then $\vec{e}_{i}=\vec{a}\left[\begin{array}{ll}I_{r} & A\end{array}\right]$ for some $\vec{a} \in R^{r}$. Hence, $\vec{a}=\overrightarrow{0}$, and so $\vec{e}_{i}=\overrightarrow{0}$, a contradiction. Thus, if $\vec{e}_{i}$ is a standard basis vector in $C$, then $1 \leq i \leq r$. Assume that $\vec{e}_{i_{1}}, \vec{e}_{i_{2}}, \ldots, \vec{e}_{i_{k}}$ are $k$ standard basis vectors in $C$ where $i_{1}, i_{2}, \ldots, i_{k}$ are pairwise distinct indices in $\{1,2, \ldots, r\}$. Since $\vec{e}_{i_{j}}$ is generated by $\left[\begin{array}{ll}I_{r} & A\end{array}\right]$, the $i_{j}^{\text {th }}$ row of $\left[\begin{array}{ll}I_{r} & A\end{array}\right]$ must be the standard basis 
vector $\vec{e}_{i_{j}}$ for any $j \in\{1,2, \ldots, k\}$. We next interchange the rows by placing rows $i_{1}, i_{2}, \ldots, i_{k}$ in the first $k$ rows. After permuting the first $r$ columns, we see that $C$ is equivalent to a free linear code generated by

$$
\left[\begin{array}{ccc}
I_{k} & 0 & 0 \\
0 & I_{r-k} & A^{\prime}
\end{array}\right]
$$

which contains at least $k$ standard basis vectors.

From Proposition 3.5, a free linear code containing exactly $k$ standard basis vectors is related to a matrix having exactly $k$ rows of single unit. This yields Corollary 3.6.

Corollary 3.6. If $C$ is a free linear code of $R^{n}$ of rank $r$ containing exactly $k$ standard basis vectors, then $C$ has a generator matrix which is of rank $r$ with $k$ rows of single unit.

Proof. Let $C$ be a free linear code of $R^{n}$ of rank $r$ containing exactly $k$ standard basis vectors. By Proposition 3.5 we may assume that $C$ is a code with a generator matrix $G=\left[\begin{array}{ccc}I_{k} & 0 & 0 \\ 0 & I_{r-k} & A^{\prime}\end{array}\right]$ where $A^{\prime}$ is an $(r-k) \times(n-r)$ matrix over $R$. Note that $\operatorname{rank} G=\operatorname{rank} \pi(G)=r$. Since $C$ does not contain $k+1$ standard basis vectors, none of the rows of $A^{\prime}$ are zero vectors. Thus, $G$ is a matrix of rank $r$ having exactly $k$ rows of single unit.

Finally, we note that, if $C$ is a free linear code of $R^{n}$ over a commutative finite local ring $R$ of rank $r$ with a basis $\left\{\vec{b}_{1}, \vec{b}_{2}, \ldots, \vec{b}_{r}\right\}$, then $\pi(C)$ is a linear code of $(R / M)^{n}$ over the residue field $R / M$ of dimension $r$ with a basis $\left\{\pi\left(\vec{b}_{1}\right), \pi\left(\vec{b}_{2}\right), \ldots, \pi\left(\vec{b}_{r}\right)\right\}$ (see [12]). From this, we derive the number of free linear codes of $R^{n}$ that contain exactly $k$ standard basis vectors.

Theorem 3.7. Let $R$ be a commutative finite local ring with maximal ideal $M$, residue field $R / M$ with $q$ elements, and the natural map $\pi: R \rightarrow R / M$.

(1) If $C$ is a free linear code of $R^{n}$ of rank $r$ containing at least $k$ standard basis vectors, then $\pi(C)$ is a linear code of $(R / M)^{n}$ of dimension $r$ containing at least $k$ standard basis vectors.

(2) A linear code of $(R / M)^{n}$ of dimension $r$ containing at least $k$ standard basis vectors can be lifted to $|M|^{(n-r)(r-k)}$ free linear codes of $R^{n}$ of rank $r$ containing at least $k$ standard basis vectors.

(3) The number of free linear codes of $R^{n}$ of rank $r$ containing exactly $k$ standard basis vectors is

$$
\sum_{\ell=k}^{r}(-1)^{\ell-k}\left(\begin{array}{l}
\ell \\
k
\end{array}\right)\left(\begin{array}{l}
n \\
\ell
\end{array}\right)|M|^{(n-r)(r-k)}\left[\begin{array}{l}
n-\ell \\
n-r
\end{array}\right]_{q} .
$$

Proof. It is easy to see that, if $\vec{e}_{i}$ is a standard basis vector in $R^{n}$, then $\pi\left(\vec{e}_{i}\right)$ is a standard basis vector in $(R / M)^{n}$. This proves (1).

For (2), let $\bar{C}$ be a linear code of $(R / M)^{n}$ of dimension $r$ containing at least $k$ standard basis vectors. By Proposition 3.5, we may assume that a generator matrix $\bar{G}$ for $\bar{C}$ is

$$
\bar{G}=\left[\begin{array}{ccc}
\bar{I}_{k} & \overline{0} & \overline{0} \\
\overline{0} & \bar{I}_{r-k} & \bar{A}
\end{array}\right],
$$

where $\bar{A}$ is an $(r-k) \times(n-r)$ matrix over $R / M$ and $\bar{I}_{k}, \bar{I}_{r-k}$ are the identity matrices over $R / M$. We write $\bar{A}=\left[a_{i j}+M\right]$ and let $A=\left[a_{i j}\right]$. Consider a linear code $C$ of $R^{n}$ generated by

$$
G=\left[\begin{array}{ccc}
I_{k} & 0 & 0 \\
0 & I_{r-k} & A+N
\end{array}\right],
$$

where $N$ is an $(r-k) \times(n-r)$ matrix, all entries of which are in $M$. Since $\operatorname{rank} G=\operatorname{rank} \pi(G)=\operatorname{rank}(\bar{G})=r, C$ is a free linear code of rank $r$. From the first $k$ rows of the matrix $G$, we see that $C$ contains at least $k$ standard 
basis vectors. Next, let

$$
\mathcal{C}(\bar{G})=\left\{\left[\begin{array}{ccc}
I_{k} & 0 & 0 \\
0 & I_{r-k} & A+N
\end{array}\right]: N \text { is an }(r-k) \times(n-r) \text { matrix whose entries are in } M .\right\} .
$$

Note that, if $C$ is a free linear code of $R^{n}$ generated by a matrix in $\mathcal{C}(\bar{G})$, then $\pi(C)=\bar{C}$. Suppose that

$$
G_{1}=\left[\begin{array}{ccc}
I_{k} & 0 & 0 \\
0 & I_{r-k} & A+N_{1}
\end{array}\right] \text { and } G_{2}=\left[\begin{array}{ccc}
I_{k} & 0 & 0 \\
0 & I_{r-k} & A+N_{2}
\end{array}\right]
$$

are two matrices in $\mathcal{C}(\bar{G})$ that generate the same code. Then $G_{1}=U G_{2}$ where $U \in G L_{r}(R)$. We can see that $U=I_{r}$. Hence, $N_{1}=N_{2}$. Thus, all matrices in $\mathcal{C}(\bar{G})$ generate distinct free linear codes. Proposition 3.5 further implies that any free linear code $C$ of $R^{n}$ of rank $r$ that contains at least $k$ standard basis vectors and where $\pi(C)=\bar{C}$ has a generator matrix of the form (3.1). Therefore, $\mathcal{C}(\bar{G})$ generates the set of $|M|^{(n-r)(r-k)}$ free linear codes of $R^{n}$ of rank $r$ containing at least $k$ standard basis vectors. These are lifted from $\bar{C}$.

We next prove (3). By the proof of Theorem 6 in [1], the number of linear codes of $(R / M)^{n}$ of dimension $r$ containing $\ell$ standard basis vectors $\vec{e}_{i_{1}}, \vec{e}_{i_{2}}, \ldots, \vec{e}_{i_{\ell}}$ is equal to $\left[\begin{array}{l}n-\ell \\ n-r\end{array}\right]_{q}$. By (2), each can be lifted to $|M|^{(n-r)(r-\ell)}$ free linear codes of $R^{n}$ of rank $r$ containing $\vec{e}_{i_{1}}, \vec{e}_{i_{2}}, \ldots, \vec{e}_{i_{\ell}}$. Note that there are $\left(\begin{array}{l}n \\ \ell\end{array}\right)$ sets of $\ell$ standard basis vectors. Hence, the number of free linear codes of $R^{n}$ of rank $r$ containing at least $\ell$ standard basis vectors is

$$
\left(\begin{array}{c}
n \\
\ell
\end{array}\right)|M|^{(n-r)(r-\ell)}\left[\begin{array}{l}
n-\ell \\
n-r
\end{array}\right]_{q} .
$$

We finally apply the Principle of Inclusion and Exclusion [9] to conclude that the number of free linear codes of $R^{n}$ of rank $r$ containing exactly $k$ standard basis vectors is

$$
\sum_{\ell=k}^{r}(-1)^{\ell-k}\left(\begin{array}{l}
\ell \\
k
\end{array}\right)\left(\begin{array}{l}
n \\
\ell
\end{array}\right)|M|^{(n-r)(r-\ell)}\left[\begin{array}{l}
n-\ell \\
n-r
\end{array}\right]_{q}
$$

as desired.

In summary, we have investigated matrices with a given number of rows of single unit and free linear codes containing a given number of standard basis vectors over commutative finite local rings. These matrices and free linear codes can be applied in coding theory. We know that an $m \times n$ matrix can be used to transmit messages of length $m$. Indeed, if $G$ is an $m \times n$ matrix, we may encode a message $\vec{u} \in R^{m}$ with a codeword $\vec{u} G$. Matrices with rows of single unit would therefore allow a message to be encoded facilely. Encoding and decoding is simplified if the code is a free linear code whose generator matrix comprises rows of single unit.

Acknowledgments: The author would like to thank the referee for the valuable comments, and Mr. John Winward for editorial suggestions.

Data Availability Statement: Data sharing is not applicable to this article as no datasets were generated or analyzed during the current study.

\section{References}

[1] Abdel-Ghaffar, K.: Counting matrices over finite field having a given number of rows of unit weight, Linear Algebra Appl. 436 (2012), 2665-2669.

[2] Bollman, D., Ramirez, H.: On the enumeration of matrices over finite commutative rings, Amer. Math. Monthly 76 (1969), 1019-1023.

[3] Brawley, J.V., Carlitz, L.: Enumeration of matrices with prescribed row and column sums, Linear Algebra Appl. 6 (1973), 165174. 
[4] Brown, W.C.: Matrices over Commutative Rings, Marcel Dekker, Inc., New York, 1993.

[5] Ching, W.S.: Linear equations over commutative rings, Linear Algebra Appl. 18 (1977), 257-266.

[6] Dougherty, S.T.: Algebraic Coding Theory over Finite Commutative Rings, SpringerBriefs in Mathematics, Springer, Cham, 2017.

[7] Dougherty, S.T., Saltürk, E.: Counting codes over rings, Des. Codes Cryptogr. 73 (2014), 151-165.

[8] Fisher, S.D., Alexander, M.N.: Matrices over a finite field, Amer. Math. Monthly 73 (1966), 639-641.

[9] Hall, M. Jr.: Combinatorial Theory, second ed., Wiley, New York, 1986.

[10] Huffman, W.C., Pless, V.: Fundamentals of Error-Correcting Codes, Cambridge University Press, Cambridge, 2013.

[11] McCoy, N.H.: Rings and Ideals, Carus Math. Monogr. No. 8, Math. Assoc. of Am., 1948.

[12] Sirisuk, S., Meemark, Y.: Generalized symplectic graphs and generalized orthogonal graphs over finite commutative rings, Linear Multilinear Algebra 67 (2019), 2427-2450.

[13] Solé, P.: Codes over Rings, Series on Coding Theory and Cryptology: Vol.6, World Scientific Publishing Co., Singapore, 2009.

[14] Wan, Z.: Geometry of Classical Groups over Finite Fields, 2nd ed., New York: Science Press, Beijing, 2002. 